\title{
Interaction between Acupuncture and Drug
}

\author{
${ }^{1}$ Department of Neurology, Changhua Christian Hospital, Taiwan \\ ${ }^{2}$ Department of Medicinal Botanicals and Health Applications, Da-Yeh University, Taiwan \\ ${ }^{3}$ Department of Surgery, Cheng-Ching Hospital, Taiwan \\ ${ }^{4}$ Department of Orthopedics, Taichung Veterans General Hospital, Taiwan \\ ${ }^{5}$ Department of Bioindustry Technology, Da-Yeh University, Taiwan \\ ${ }^{6}$ School of Chinese Medicine, China Medical University, Taiwan
}

ChihMing Lin ${ }^{1,2}$, YuanChiang Chung ${ }^{2,3}$, ChungYuh Tzeng ${ }^{4}$, YingI Chen ${ }^{2}$, TaiHao Hsu ${ }^{5}$ and ShihLiang Chang*2,

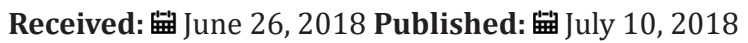

*Corresponding author: ShihLiang Chang, No.168, University Rd, Changhua County, Taiwan

\begin{abstract}
Can the acupuncture stimulations impact on the effect or lower the dose of drugs stimulation using at the same time and the same organism? In recent years, we applied electroacupuncture (EA), a physical stimulation and some drugs (insulin, hypoglycemic agents, steroids or volatile anesthetics. etc.) at the same time on the same animal model to test the hypoglycemic activity obtain that the EA stimulation may elevated the hypoglycemic effect of drugs. In another physical stimulation, Ear acupoints were stimulated by small balls obtained that lower down the effect dose of atropine for the treatment of myopia. There were some interactions such as: antagonism, synergism, potentiation and summation. etc. This is an important issue worth for further investigations.
\end{abstract}

Keywords: Interaction; Electroacupuncture; Hypoglycemic agent; Steroid; Volatile Anesthetics

Abbreviations: EA: Electro Acupuncture; TENS: Transcutaneous electrical Nerve stimulation; STZ: Streptozotocin; FFA: Free Fatty Acid; MAPK: Activating Mitogen-activated protein kinase; SIIR: Steroid Induced Insulin Resistant

\section{Introduction}

As we know there are some interactions between two drugs named drug-drug interaction. One drug can be changed the effect of the other drug which is applied before or at the same time with the other drug [1]. The bad drug interaction may change of the pharmacological activity to induce lower effect of drug, even to archive over dose or toxicity [2]. But, well drug interaction may archive better effect than used alone, lower down the effective dose or against the disadvantages. There are several types of drug interaction such as, antagonism, synergism, potentiation and summation etc [3]. The mechanisms of drug interaction may due to the change of pharmacokinetic such as absorption, distribution, metabolism and excretion [4]. To answer a question, can the acupuncture stimulations impact on the effect or lower the dose of drugs stimulation, at the same time and the same organism? Several our previous studies will be discussed and try to answer this question [5-8]. Acupuncture, electroacupuncture (EA) or transcutaneous electrical nerve stimulation (TENS) are the method of physical stimulations on the acupoint that would change the effect or their disadvantage effect of drugs (insulin, hypoglycemic agents, steroids or volatile anesthetics..etc) [6-12].

\section{EA Enhanced the Hypoglycemic Effect of Insulin}

A physical stimulation, EA and exogenous regular insulin, which the hypoglycemic onset is $30 \mathrm{~min}$, were used on the Normal rat and Streptozotocin (STZ)-induced diabetic rat at the same time and compare with non-EA group, only injecting regular insulin in 2006 [6] .The more hypoglycemic activity in bilateral Zusanli acupoint (ST36) EA group was obtained than in the non-EA group $30 \mathrm{~min}$ after the start of insulin injecting to the normal rat. Also, 60 minutes after the start of insulin injection, the hypoglycemic activity of the EA group still was significantly higher than that of the non-EA group. The STZ-induced diabetic rats showed the same trends as the normal rats, including that more hypoglycemic activity for the EA group was obtained than for the non-EA group[6]. In this case, the physical stimulation, EA may enhance the hypoglycemic activity of regular insulin and/or insulin sensitivity that would be thought as a summation or synergism or potentiation effect.

\section{EA Enhance the Hypoglycemic Effect of Hypoglycemic Agents}

There were some kinds of hypoglycemic agents such as rosiglitazone, metformine..etc using combined with EA for observing the interaction on the hypoglycemic effect [7-11]. In 2009, the hypoglycemic effect of rosiglitazone was increased by EA in both normal and type 2 diabetic rats, indicating that the application of EA may enhance the hypoglycemic action of this insulin sensitizer. Also, a randomized control clinical trial was reported to reveal this interaction between EA and rosiglitazone in 
2013. This combined therapy seems to suppress endogenous insulin secretion of type 2 diabetic patient by improving insulin resistance via a reduction in plasma free fatty acid (FFA). Until 2015, a report entitled "Electroacupuncture plus metformin lowers glucose levels and facilitates insulin sensitivity by activating mitogen-activated protein kinase (MAPK) in steroid-induced insulin-resistant rats" showed that this combined therapy resulted in a greater glucoselowering effect, higher insulin sensitivity, lower plasma FFA levels and higher levels of MAPK than metformin alone [11]. This study also showed a physical stimulation, EA enhance the hypoglycemic effect of hypoglycemic drug that would be thought as a summation or synergism or potentiation effect.

\section{EA Improved the Insulin Resistance Induced by Steroid}

The plasma glucose level is elevated by archiving insulin resistance usually that is disadvantage effect of steroid treatment. In 2009, a study entitled "Acute effect of electroacupuncture at the Zusanli acupoints on decreasing insulin resistance as shown by lowering plasma free fatty acid levels in steroid-background male rats" that reported the steroid induced insulin resistant (SIIR) state can be improved by $15 \mathrm{~Hz}$ EA at the bilateral Zusanli acupoints, as shown by decreased plasma levels of FFA $[12,13]$. Also, another study reported that EA decreased the FFA level and increased insulin sensitivity in dexamethasone chronic induced insulin resistant rats in 2016 [12]. The EA stimulation lowered down plasma FFA level and enhanced the insulin signal transduction of SIIR. EA improved the insulin resistance induced by steroid that would be a style of interaction, diminish their disadvantage effect of drugs.

\section{TENS Improved the Insulin Resistance Induced by Volatile Anesthetics}

Volatile anesthetics directly manipulate glucose homeostasis by affecting pancreatic insulin release and induce hyperglycemia usually that is disadvantage effect of anesthesia during surgery especially in hyperglycemic patient. In 2011, a physical stimulation, TENS stimulated to the Chinese acupoints Zusanli (ST36) and Sanyinjiao (SP6) of patient before surgery as a complementary therapy for controlling plasma glucose and improving insulin resistance during anesthesia. This study concluded that the TENS at bilateral ST36 and SP6 acupoints to be an alternative means of managing the plasma glucose level and improving insulin resistance [8]. TENS improved the insulin resistance induced by volatile anesthetics that would be a style of interaction, diminish their disadvantage effect of drugs.

\section{Auricular acupoints stimulation reduce the effective dose of atropine for treating myopia}

In 2008, a randomized single-blind clinical controlled trial was designed to compare the reduction in myopia progression in patients treated with atropine eye drops alone with patients treated with a combined treatment of atropine and stimulation of the auricular acupoints. The school-aged children with myopia were randomly assigned into three groups. These were (1) $0.25 \mathrm{~A}$ : treated with the $0.25 \%$ atropine only, (2) $0.5 \mathrm{~A}$ : treated with the $0.5 \%$ atropine only and (3) $0.25 \mathrm{~A}+\mathrm{E}$ : treated with $0.25 \%$ atropine together with stimulation of the auricular acupoints. This study demonstrates that there was efficacy in stimulating the auricular acupoints and this enhanced the action of $0.25 \%$ atropine as a means of myopia control. The result was an effect almost equal to that of $0.5 \%$ atropine alone [14]. Auricular acupoints stimulation reduced the effective dose of atropine for treating myopia that would be thought as a synergism or potentiation effect.

\section{Conclusion}

Taken together, the physical stimulations such as TENS, EA or acupuncture impact on the effect or lower the effective dose of drugs stimulation using at the same time and the same organism. That may have interaction between acupuncture and drugs. This issue is worthy systemic exploration.

\section{Acknowledgements}

The authors would like to thank the supportive grants. This study was supported by grants provided by the Ministry of Science and Technology (MOST-106-2622-E-212-002-CC2), Taichung Veterans General Hospital and Da-Yeh University joint project (TCVGH-DYU-107-8301), and Cheng Ching Hospital and Da-Yeh University joint project (CCGH-DYU-106-001) in Taiwan.

\section{References}

1. Ibanez A (2008) Drug-drug interactions in patients from an internal medicine service. Farm Hosp 32(5): 293-297.

2. Nalamachu S, Pergolizzi JV, Raffa RB, Lakkireddy DR, Taylor R, et al. (2004) Drug-drug interaction between NSAIDS and low-dose aspirin: a focus on cardiovascular and GI toxicity. Expert Opin Drug Saf 13(7): 903-917.

3. Pöch G (1993) Synergism/potentiation and antagonism ??phenomena and mechanisms, in Combined Effects of Drugs and Toxic Agents: Modern Evaluation in Theory and Practice. Springer Vienna Vienna p. 39-53.

4. Vugmeyster YJ, Harrold X, Xu (2012) Absorption, distribution, metabolism, and excretion (ADME) studies of biotherapeutics for autoimmune and inflammatory conditions. AAPS J 14(4): 714-727.

5. Chang SL, Lin JG, Chi TC, Liu IM, Cheng JT, et al. (1999) An insulindependent hypoglycaemia induced by electroacupuncture at the Zhongwan (CV12) acupoint in diabetic rats. Diabetologia 42(2): 250255.

6. Chang, SL, Lin KJ, Lin RT, Hung PH, Lin JG, et al. (2006) Enhanced insulin sensitivity using electroacupuncture on bilateral Zusanli acupoints (ST 36) in rats. Life Sci 79(10): 967-971.

7. Lin RT, Pai HC, Lee YC, Tzeng CY, Chang CH, et al. (2013) Electroacupuncture and rosiglitazone combined therapy as a means of treating insulin resistance and type 2 diabetes mellitus: a randomized controlled trial. Evid Based Complement Alternat Med pp. 969824.

8. Man KM, Man SS, Shen JL, Law KS, Chen SL, et al. (2011) Transcutaneous electrical nerve stimulation on ST36 and SP6 acupoints prevents hyperglycaemic response during anaesthesia: a randomised controlled trial. Eur J Anaesthesiol 28(6): 420-426.

9. Pai HC, Tzeng CY, Lee YC, Chang CH, Lin JG, et al. (2009) Increase in plasma glucose lowering action of rosiglitazone by electroacupuncture at bilateral Zusanli acupoints (ST.36) in rats. J Acupunct Meridian Stud 2(2): 147-151.

10. Lin RT (2014) Acupoint-specific, frequency-dependent, and improved insulin sensitivity hypoglycemic effect of electroacupuncture applied to drug-combined therapy studied by a randomized control clinical trial. Evid Based Complement Alternat Med pp. 371475. 
11. Liao HY, Sun MF, Lin JG, Chang SL, Lee YC, et al. (2015) Electroacupuncture plus metformin lowers glucose levels and facilitates insulin sensitivity by activating MAPK in steroid-induced insulin-resistant rats. Acupunct Med 33(5): 388-394.

12. Tzeng CY, Lee YC, Chung JJ, Tsai JC, Chen YI, et al. (2016) $15 \mathrm{~Hz}$ electroacupuncture at ST36 improves insulin sensitivity and reduces free fatty acid levels in rats with chronic dexamethasone-induced insulin resistance. Acupunct Med 34(4): 296-301.

\section{ISSN: 2574-1241}

DOI: $10.26717 /$ BJSTR.2018.06.001379

ShihLiang Chang. Biomed J Sci \& Tech Res

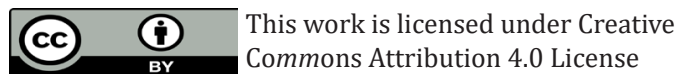

Submission Link: https://biomedres.us/submit-manuscript.php
13. Lin RT, Chung Yuh Tzeng, Yu Chen Lee, Wai Jane Ho, Juei Tang Cheng, et al. (2009) Acute effect of electroacupuncture at the Zusanli acupoints on decreasing insulin resistance as shown by lowering plasma free fatty acid levels in steroid-background male rats. BMC Complement Altern Med 9: 26

14. Cheng HC, YT Hsieh (2014) The effect of low-concentration atropine combined with auricular acupoint stimulation in myopia control. Complement Ther Med 22(3): 449-455.

Assets of Publishing with us
BIOMEDICAL
RESEARCHES

